Classification

Physics Abstracts 61.30

\title{
BOND ORIENTATIONAL ORDER MODEL FOR SMECTIC B LIQUID CRYSTALS
}

\author{
R. J. BIRGENEAU (*) and J. D. LITSTER (**) \\ Department of Physics, Massachusetts Institute of Technology, \\ Cambridge, Massachusetts, 02139, U.S.A.
}

(Reçu le 27 juin 1978, révisé le 8 septembre 1978, accepté le 11 septembre 1978)

\begin{abstract}
Résumé. - Nous utilisons les concepts pris dans les théories récentes sur la fusion des solides à 2 dimensions pour définir les paramètres d'ordre des phases solide, smectiques $\mathbf{A}$ et $\mathbf{B}$ des cristaux liquides. Dans les phases smectiques bien ordonnées, de type B par exemple, nous introduisons, un ordre à longue distance entre les orientations des liaisons des molécules proches voisines en plus de l'ordre de position à courte distance dans le plan des couches. Les implications de ce modèle pour la diffusion des rayons $\mathrm{X}$ sont discutées en détail.
\end{abstract}

\begin{abstract}
We show how recent theories of two dimensional melting can be carried over to provide natural order parameters for solid, smectic B, and smectic A phases of liquid crystals. Smectic B and other well-ordered smectic phases correspond to systems with bond orientational long range order and positional short range order in the plane of the smectic layers. The X-ray scattering predicted by this model is discussed in detail.
\end{abstract}

Recently smectic liquid crystals, which have long range orientational order of the molecules combined with varying degrees of positional order, have been the subject of extensive experimental and theoretical investigation. As a result we have rather good theoretical models for the smectic A (SmA) and smectic $\mathrm{C}(\mathrm{SmC})$ phases characterized by one dimensional density waves whose wave vector is along ( $\mathrm{SmA})$ or at an angle $(\mathrm{SmC})$ to the nematic director [1]. Also, as a result of combined light and high resolution X-ray scattering, a rather good quantitative experimental description of the SmA phase is now emerging [2]. One of the more interesting features of the SmA phase is that the lower marginal dimensionality $d^{0}$ is three [3]; this is the spatial dimensionality at which fluctuations prevent the establishment of true long range order $[4,5,6]$. Between the $\mathrm{SmA}$ or $\mathrm{SmC}$ phases and the crystalline solid of many materials are one or more intermediate phases which are characterized by a considerable degree of order within and between the smectic layers. Although beautiful experimental

(*) Supported by Joint Services Electronics Program, contract $\mathrm{n}^{\circ}$ DAAG-29-78-C-0020.

(**) Supported by the National Science Foundation, grant no DMR-76-18035. work has been done on the $\mathrm{SmB}$ and similar phases, especially by the Orsay group [7,8], little progress has been made on the theoretical front. In particular no convincing microscopic definition has been given for the order parameter(s) of these phases to distinguish them from the SmA or SmC phases and the crystalline solid.

Marginal dimensionality in ordinary solids occurs at $d^{0}=2$ and considerable theoretical work has been carried out for two dimensional (2D) solids [9, 10]. In this paper we suggest how the concepts introduced by Halperin and Nelson (HN) [10] for the 2D melting problem can be carried over to 3D liquid crystals. We emphasize that our discussion is essentially qualitative in nature. However, this 2D melting analogy appears to be very successful in accounting for the overall features of smectic liquid crystals. We hope therefore that our observations will serve to inspire more rigorous theory for liquid crystals of the sort carried out by $\mathrm{HN}$ for 2D melting.

We begin with a summary of the experimentally known facts for the various smectic phases. Most of these, especially with respect to macroscopic properties, have been discussed elsewhere $[1,11]$, and we limit our attention to X-ray scattering patterns. In the $\mathrm{SmA}$ and SmC phases the distribution of molecules 
in the planes normal to the density wave is random as in a conventional liquid ; the X-ray powder pattern [12] shows a sharp ring at $q_{0}=2 \pi / d$, where $d$ is the density wave period, and a diffuse outer ring corresponding to the first peak in the in-plane liquid structure factor. In the phases between SmA or SmC and the crystalline solid the diffuse outer ring becomes a series of sharp rings, reminiscent of the rings in a crystalline powder pattern $[7,8,11]$, but with several essential differences. First of all, only a small number of primary reflections are observed. In multidomain $\mathrm{SmB}$ phases often only the primary (110) in plane reflections are clearly visible, while in $\mathrm{SmH}$ or $\mathrm{SmE}$ phases one can typically resolve (110), (111), (200), (201), (210), and (211) reflections. The important observation is that only reflections with small Miller indices are clearly visible, and this has led most workers to describe these SmB like phases as ones with considerable local order or quasi-long range order, but without a precise definition of the latter concept. A second feature, especially for SmB's, is that the observable reflections are accompanied by very pronounced diffuse scattering whose intensity varies as $\Delta q^{-2}=|\mathbf{q}-\mathbf{G}|^{-2}$, where $\mathbf{q}$ is the momentum transfer and $\mathbf{G}$ is a reciprocal lattice position. The third feature, and most important from our point of view, is that one can prepare single domain samples in which one observes, for example, six well defined (200) or equivalent reflections rather than a ring of scattering. This feature precludes any model based on short range order (S.R.O.) alone.

A possible association between $\mathrm{SmB}$ liquid crystals and 2D melting was first noticed by de Gennes and Sarma [13]. Explicitly they considered a set of idealized 2D harmonic systems stacked to yield a 3D structure. Since de Gennes and Sarma proposed their model considerable progress has been made in the theory of 2D melting. In particular it is now believed that the harmonic model does not provide a realistic picture of melting and that for an isotropic $2 \mathrm{D}$ model melting occurs as a result of the disassociation of bound pairs of point dislocations - this is the Kosterlitz-Thouless mechanism [9]. However, in a crystal the orientational anisotropy, such as the six-fold symmetry of a triangular or hexagonal lattice, must be explicitly considered in the theory. Such a theory has recently been given by Halperin and Nelson (HN); they point out that one must consider both positional and orientational correlation functions. The positional order may be quantitatively described by the correlation function

$$
P(\mathbf{G}, \mathbf{r})=\left\langle\mathrm{e}^{i \mathbf{G} \cdot[\mathbf{u}(\mathbf{r})-\mathbf{u}(0)]}\right\rangle
$$

where $\mathbf{u}(\mathbf{r})$ is the displacement of the lattice at $\mathbf{r}$. The orientational order parameter [14] for six-fold symmetry is defined by $\psi(\mathbf{r}) \equiv \mathrm{e}^{i \epsilon \theta(\mathbf{r})}$ where $\theta$ specifies the orientation of a bond (i.e. the line between the centres of mass) between two nearest neighbour molecules at $\mathbf{r}$ (see Fig. 1). Then the orientational

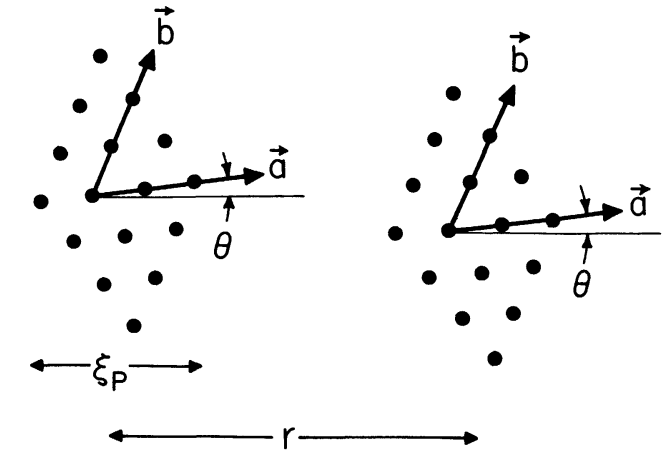

Orientational L.R.O., Positional S.R.O.

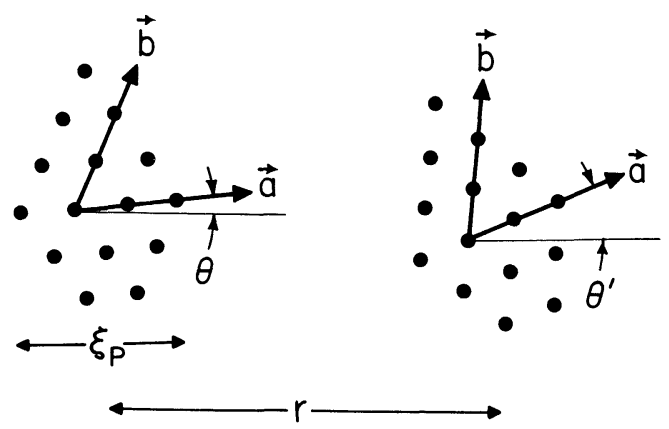

Orientational S.R.O., Positional S.R.O.

FIG. 1. - Correlated triangular droplets in state (ii), upper, and state (iii), lower, of the Halperin Nelson model for two dimensional melting. Here $d$ is the separation between the droplets and $\xi_{\mathrm{p}}$ is the positional correlation length within the droplets.

correlation function is

$$
O(\mathbf{r})=\left\langle\psi^{*}(\mathbf{r}) \psi(0)\right\rangle
$$

Mermin has shown rigorously [14] for an ideal 2D system that the positional order parameter $\left\langle\mathrm{e}^{i \mathbf{G} . \mathbf{u}}\right\rangle$ is zero at all finite temperatures, but that a $2 \mathrm{D}$ solid may have bond orientational long range order (L.R.O.) since $O(\mathbf{r})$ does not decay to zero at large $r$.

Halperin and Nelson have shown by explicit calculation with an elastic continuum Hamiltonian that a 2D crystal has three distinct phases. These are phase (i), at low temperatures with orientational L.R.O. of near neighbour bonds and algebraic decay of position order, so that

$$
P(\mathbf{G}, \mathbf{r}) \sim r^{-\eta_{G}(T)} ; \quad O(\mathbf{r}) \sim\langle\psi\rangle^{2} .
$$

At intermediate temperatures is phase (ii) with positional short range order and algebraic decay of orientational correlations, thus

$$
P(\mathbf{G}, \mathbf{r}) \sim \frac{\mathrm{e}^{-r / \xi_{\mathbf{p}}}}{r^{1 / 2 \cdot}} ; \quad O(\mathbf{r}) \sim r^{-\eta_{6}(T)}
$$

In phases (i) or (ii) the algebraic decay of correlation functions is accompanied by an infinite corresponding 
susceptibility. The high temperature phase (iii) has only short range order with

$$
P(\mathbf{G}, \mathbf{r}) \sim \frac{\mathrm{e}^{-r / \xi_{\mathrm{p}}}}{r^{1 / 2}} ; \quad O(\mathbf{r}) \sim \frac{\mathrm{e}^{-r / \xi_{o}}}{r^{1 / 2}}
$$

We suggest the following heuristic application of these results to $\mathrm{SmB}$ and similar phases. Analogous to de Gennes and Sarma, we identify the solid phase with stacked layers of HN's phase (i). The infinite positional susceptibility means that an infinitesimal interaction between layers will convert the algebraic decay of positional correlation functions into true 3D long range order. For this same reason it seems highly unlikely that the SmB phase can be formed of stacked Kosterlitz-Thouless quasi-ordered 2D solids, as was recently proposed by Huberman, Lublin, and Doniach [15]. In the 2D harmonic system the positional susceptibility is finite above a certain temperature so that layers may be stacked to form a SmB phase in the de Gennes Sarma model.

We propose that the SmB and higher ordered smectic phases can be described as stacked layers of $\mathrm{HN}$ phase (ii). We expect that interactions between layers will cause true in-plane bond orientational L.R.O. rather than the algebraic decay of $O(\mathbf{r})$ in $\mathrm{HN}$ phase (ii). Since the SmA phase has algebraic decay of positional correlations [16] along the 1D density wave and by analogy with 2D crystals has bond orientational L.R.O. in the same direction, the SmB phase should have true $3 \mathrm{D}$ bond orientational long range order. Since the positional in-plane correlations of phase (ii) decay exponentially, the corresponding susceptibility will be finite. Thus we expect stacked layers of phase (ii) can exist as well-ordered smectic phases with layers free to slide on one another provided the between layer interactions are sufficiently weak. The melting of the aliphatic chains [17] would provide a mechanism to reduce the interaction, which should be weaker for longer chains; this probably explains why only the longer chain members of a homologous series exhibit SmB like phases.

Finally, we identify stacked layers of $\mathrm{HN}$ phase (iii) with $\mathrm{SmA}$ or SmC phases. As mentioned above, the layers are stacked with algebraic decay of the positional correlations along the 1D density wave directions. The bond orientational L.R.O. which is present by analogy with 2D solids manifests itself as the fixed angle between the molecular axes and the smectic layers. The states of our model are summarized in table I.

\section{TABLE I}

\section{Correlations in liquid crystal phases}

$\begin{array}{lc}\text { Phase } & \text { In-plane bond orientational } \\ \text { correlations }\end{array}$

In-plane positional correlations L.R.O.

Exponential decay (S.R.O.)

Exponential decay (S.R.O.)
There are further subdivisions to be made to describe the various well-ordered smectic phases; these include tilt angles, rotational degrees of freedom about molecular long axes, and the type of in-plane lattice. In the tilted phases, the molecular tilt means twofold orientational in-plane L.R.O. even in the $\mathrm{SmC}$ phase [18] which will induce some six-fold order. Thus the well-ordered tilted phases differ from SmC in that the bond orientational L.R.O. is a primary order parameter rather than induced. Most of the discussion in the literature [e.g. 8] can be incorporated directly into our bond orientational L.R.O. model.

We now discuss some of the consequences of our model. Clearly the primary consequence is the identification of a microscopic order parameter which enables a quantitative differentiation between $\mathrm{SmA}$, $\mathrm{SmC}$, and the well-ordered SmB type phases.

The model also suggests a physical explanation for the strange temperature dependence of the SmA phase elastic constants $B$ and $D$ [19]. These constants give, respectively, the restoring force for compression of the smectic layers and for tilt of the molecules away from perpendicular to the layers. Several experiments have established $[19,20,21]$ that $B$ vanishes at the SmA-nematic transition with a power law quite different from that predicted by analogy with 3D superfluid helium; we believe this to be the result of the divergent phase fluctuations of the SmA order parameter and the absence of true positional L.R.O. In addition, $D$ goes to zero with a different power law than $B[19,2]$, a result quite unexpected from the ${ }^{4} \mathrm{He}$ analogue. In our view, since $D$ is directly associated with the angle between the molecules and the smectic layers, which has true L.R.O., we might expect different critical behaviour for $D$ compared with $B$.

To begin a discussion of the implications of our model for X-ray diffraction patterns, we remind the reader that the results of an X-ray experiment are sensitive to the positions of the molecules, and that one normally observes peaks in the scattered intensity at momentum transfers corresponding to reciprocal lattice vectors. Our model for the $\mathrm{SmA}$ and $\mathrm{SmC}$ phases is identical to the conventional one, a 1D density wave with algebraic decay of positional correlations together with a weakly correlated liquid transverse to the density wave direction. Hence we predict the usual X-ray pattern.

For SmB type phases important differences occur. The in-plane orientational L.R.O. makes it possible to prepare single domain samples with well defined bond orientations across the entire sample. The effect is illustrated in figure 1. Instead of the ring one would observe with orientational S.R.O., for a single domain sample with orientational L.R.O. we predict resolved peaks at the reciprocal lattice positions of the inplane lattice (hexagonal for $\mathrm{SmB}$ ). We also discuss the shape of these peaks. Halperin and Nelson compare their phase (ii) to a $2 \mathrm{D}$ nematic; this would have very short positional correlation lengths even in the pre- 
sence of orientational L.R.O. It seems clear however that in order to have six-fold in-plane orientational L.R.O. combined with algebraic decay of positional correlations perpendicular to the planes, there must be positional correlations that extend over quite large distances. Thus we expect the correlation length $\xi_{\mathrm{p}} \gg$ in-plane nearest neighbour separation; in $\mathrm{HN}$ phase (ii) $\zeta_{\mathrm{p}}$ ranges from $\sim 6$ lattice parameters to $\infty$ as the melting transition is approached. We therefore predict an X-ray pattern with well defined Lorentzian peaks (with $\Delta q^{-2}$ tails) centred about the lower order reciprocal lattice positions in the plane. The width of the peaks provides a measure of $\xi_{p}$ and as the in-plane order increases more reflections will become observable.

These predictions are consistent with the available information in the literature $[7,8,11,12]$. Studies so far have been carried out with relatively low resolution; ultrahigh resolution studies, similar to those of Als-Nielsen et al. [3], are required to provide a definitive test of our model. The de Gennes Sarma model is probably incorrect to the extent it is based on a 2D harmonic system, but an experimental distinction between their model and ours is possible. Our model predicts an analytic structure factor corresponding to vectors of the in-plane reciprocal lattice of the SmB phase while the de Gennes Sarma picture predicts a cusp. Finally, the de Gennes Sarma model does not provide a natural transition between the SmB and SmA phases, whereas this transition, HN phase (ii) to phase (iii), is an essential feature of our model.

In conclusion, we note that if our adaptation of the Halperin-Nelson 2D melting picture to smectic liquid crystals is correct, then smectics in turn provide a rich laboratory for investigation of the ideas developed for systems whose marginal dimensionality $d^{0}$ is two. Primarily because of substrate difficulties it is difficult to imagine, for example, a real physical 2D fluid in state (ii). However, smectics may be studied in bulk without serious substrate problems and may be probed with a wide variety of experimental tools. Thus smectic phases may provide important insights into the problem of melting in two dimensions.

Acknowledgments. - We should like to thank B. Halperin, M. Kaplan and D. Nelson for stimulating conversations about this work, and P. G. de Gennes for important comments on the manuscript.

\section{References}

[1] De Gennes, P. G., The Physics of Liquid Crystals (Oxford University Press) 1974.

[2] Litster, J. D., Birgeneau, R. J., Als-Nielsen, J., Davidov, D., Dana, S. S., Garcia-Golding, F., Kaplan, M., Safinya, C. R. and Schaetzing, R., paper presented at International Conference on Liquid Crystals, Bordeaux, 1978, to be published in J. Physique Colloq.

[3] Als-Nielsen, J., Birgeneau, R. J., Kaplan, M., Litster, J. D. and Safinya, C. R., Phys. Rev. Lett. 39 (1977) 1668.

[4] Peierls, R. E., Helv. Phys. Acta 7 (1934) 81.

[5] Landau, L. D. and Lifshitz, E. M., Statistical Physics, (Addison Wesley, Reading, Mass.) 1969, p. 402.

[6] De Gennes, P. G., J. Physique Colloq. 30 (1969) C4-65.

[7] Doucet, J., Levelut, A. M., Lambert, M., Phys. Rev. Lett. 32 (1974) 301.

[8] Levelut, A. M., Doucet, J. and Lambert, M., J. Physique 35 (1974) 773 ; Doucet, J., Levelut, A. M., Lambert, M. and Strzelecki, L., ibid 36 (1975) Cl-13; Doucet, J., Keller, P., Levelut, A. M. and Porquet, P., ibid 39 (1978) 548.
[9] Kosterlitz, J. M. and Thouless, D. J., J. Phys. C 6 (1973) 118 ; Prog. Low Temp. Phys. (to be published).

[10] Halperin, B. I. and Nelson, D. R., to be published.

[11] De VRies, A. and Fishel, D. L., Mol. Cryst. Liq. Cryst. 16 (1972) 311.

[12] De VRIES, A., J. Physique Colloq. 36 (1975) C1-1 gives a review of the literature up to 1975.

[13] De Gennes, P. G. and Sarma, G., Phys. Lett. 38A (1972) 219.

[14] Mermin, N. D., Phys. Rev. 176 (1968) 250.

[15] Huberman, B. A., Lublin, D. M. and Doniach, S., Solid State Commun. 17 (1975) 485.

[16] Caillé, A., C.R. Hebd. Séun. Acad. Sci. 274B (1972) 891 ; eq. (9) is in error, see [1], p. 298.

[17] Poldy, F., Dvolaitzky, M. and Taupin, C., J. Physique Colloq. 36 (1975) Cl-27.

[18] HalPeRIN, B. I., private communication.

[19] Birecki, H., SChaetzing, R., Rondelez, R. and Litster, J. D., Phys. Rev. Lett. 36 (1976) 1376.

[20] Ribotta, R., C.R. Hebd. Séan. Acad. Sci. 279B (1974) 295.

[21] Clark, N. A., Phys. Rev. 14A (1976) 1551. 